\title{
PARAMETRIZAÇÃO DO MÉTODO DO PERFIL INSTANTÂNEO PARA A DETERMINAÇÃO DA CONDUTIVIDADE HIDRÁULICA DO SOLO EM EXPERIMENTOS COM EVAPORAÇÃO(1)
}

\author{
Ana Lúcia Berretta Hurtado ${ }^{(2)}$, Rogerio Cichota(3) \\ $\&$ Quirijn de J ong Van Lier(4)
}

\begin{abstract}
RESUMO
A condutividade hidráulica do solo é uma propriedade cuja quantificação é essencial para qualquer estudo que envolva o movimento da água no solo. Os métodos para sua determi nação baseados na drenagem interna, como o do perfil instantâneo - MPI, são os mais empregados, restringindo-se à determinação da condutividade hidráulica entre a condição de solo saturado e a umidade na capacidade de campo. Com a finalidade de obter a condutividade hidráulica para menores valores de umidade, propôs-se um método de emprego do MPI com evaporação, apresentando-se o procedi mento para a determi nação do plano de fluxo zero e esti mativa da densidade de fluxo. Demonstrou-se a aplicação do método, obtendo-se resultados de condutividade hidráulica para a camada superficial de um perfil monitorado a partir de um experimento de campo realizado durante um período de $\mathbf{1 2}$ dias. A profundidade máxima do plano de fluxo zero estimado foi de aproximadamente $0,50 \mathrm{~m}$. A faixa de umidade obtida variou de 0,25 a $0,21 \mathrm{~m}^{3} \mathrm{~m}^{-3}$, valores complementares aos obtidos em experimentos sem evaporação no mesmo local. Os resultados indicaram a viabilidade do método, útil especial mente para se estender à faixa de umidade obti da na camada superficial de tais experimentos.
\end{abstract}

Termos de indexação: plano de fluxo zero, drenagem, ascersão.

\footnotetext{
(1) Recebido para publicação em outubro de 2003 e aprovado em janeiro de 2005.

(2) Doutoranda em Agronomia pelo Programa de Pós-Graduação em I rrigação e Drenagem da Universidade de São Paulo - ESALQ/ USP, Caixa Postal 9, CEP 13418-900 Piracicaba (SP). E-mail: alob@usp.br

(3) Doutorando em Ciência do Solo, Massey University, Palmerston North, Nova Zelândia. E-mail: RCichota@hortresearch.co.nz

(4) Professor do Departamento de Ciências Exatas, ESALQ/USP. E-mail:qdjvlier@esalq.usp.br
} 


\title{
SUMMARY: PARAMETERIZATION OF THE INSTANTANEOUS PROFILE METHOD TO DETERMINE SOIL HYDRAULIC CONDUCTIVITY IN EVAPORATION EXPERIMENTS
}

\begin{abstract}
Soil hydraulic conductivity is an essential property and its quantification is essential for any study that invol ves soil water movement. Methods of determination based on internal drainage, such as the instantaneous profile method (I PM) are among the most commonly used, but their determination is restricted to the soil water range between saturation and fied d capacity. In order to obtain hydraulic conductivity for lower water contents, wepropose an IPM that takes into account evaporation as well as a calculation routines to determine the zero flux depth and to estimate flux densities. The application of the method is demonstrated by obtaining results for hydraulic conductivity of the surface layer of a monitored profile in a 12 -day field experiment. The estimated maximum zero flux depth was about $0.50 \mathrm{~m}$. Soil water content varied from 0.25 to $0.21 \mathrm{~m}^{3} \mathrm{~m}^{-3}$, complementing the range obtained in experiments without evaporation at the same site. Results confirm the applicability of the method, which is especially useful to extend the range of soil water contents in the surface layer obtained in this kind of experiment.
\end{abstract}

Index terms: zero flux plante, drainage, ascersion.

\section{NTRODUÇÃO}

As propriedades que determinam a densidade do fluxo deágua no sol o são a condutividade hidráulica, que mede a habilidade do solo em conduzir água, e as características de retenção de água no solo, que expressam a sua habilidade em armazenar água (Klute \& Dirksen, 1986). Assim, o conhecimento da condutividade hidráulica do solo é essencial para qualquer estudo que envolva o movimento da água no solo, seja para estudar a própria dinâmica da água, seja para estudar o transporte de elementos químicos, nutrientes e defensivos agrícolas, bem como seus impactos potenciais ao ambiente.

A condutividade hidráulica $\mathrm{K}$ tem seu valor máximo no sol o saturado e éaltamente dependente da umidade $\theta$. O valor deK decresceacentuadamente com a diminuição de $\theta$ e, por esse motivo, em geral, expressa-se $K$ em função de $\theta(K(\theta))$. Alternativamente, pode-se também escrever $\mathrm{K}$ em função do potencial matricial $\psi(\mathrm{K}(\psi))$.

Além de variar com a umidade, detecta-se alta variabilidade espacial da condutividade hidráulica no solo, tanto em determinações no campo como no laboratório (Warrick \& Nielsen, 1980; J ong van Lier \& Libardi, 1999). Os métodos de campo empregados com mais sucesso para sua medição são fundamentados em experimentos de drenagem interna, como o método do perfil instantâneo - MPI - (Hillel et al., 1972), que se baseia na análise dos componentes da equação de Richards em perfis de potenciais e fluxos transientes durante a drenagem vertical que ocorre após a saturação do solo por uma chuva ou irrigação (Paige \& Hillel, 1993).

O MPI apresenta como vantagem principal a possibilidade de se medir diretamente no campo as propriedades hidráulicas, sem a necessidade de aguardar o estabel ecimento de um equilíbrio dinâmico ("steady state"). Vários autores, comparando resultados do MPI com os de outros métodos, inclusive de laboratório, concluíram que o MPI in situ resulta em determinações mais consistentes, além de sua relativa facilidade de realização (Prevedello et al., 1981; Paige \& Hillel, 1993; Marion et al., 1994), chegando a considerá-lo o método padrão.

Entretanto, o método apresenta algumas limitações. Baker et al. (1974) ePaige \& Hillel (1993) ressaltam que a drenagem interna pode ser influenciada pela presença da água subterrânea quando esta se encontra próxima à superfície; em perfis de solos heterogêneos, não há uma redistribuição de água uniforme, pois a presença de camadas menos permeáveis impede o fluxo vertical de água, favorecendo o fluxo lateral; e solos com inclinação não permitem uma inundação e uma drenagem vertical homogênea. Contudo, Shouse et al. (1992) aplicaram o método do perfil instantâneo em um perfil vertical mente heterogêneo, utilizando o escal onamento das propriedades hidráulicas, e obtiveram bons resultados.

Um dos procedimentos experimentais comuns do MPI inclui a cobertura da parcela experimental com Iona plástica, o que foi sugerido primeiramente por Ogata \& Richards (1957), evitando, assim, a evaporação ou a reaplicação de água, facilitando a análise dos dados. Posteriormente, Watson (1966) melhorou essa técnica, tornando o método mais preciso. Dessa forma, a única perda de água é por drenagem, e não se forma uma zona de fluxo ascendente no perfil. Alguns rotei ros para processar manualmente os dados desses experimentos estão disponíveis em Libardi (2000). Também podem ser encontradas rotinas computacionais, como o software Kteta (J ong van Lier \& Libardi, 1999). 
Os experimentos com cobertura de lona plástica somente permitem medir a condutividade hidráulica entre a condição saturada e a capacidade de campo, tornando-se, após essa faixa de umidade, o movimento muito lento para ser detectado (Bouma, 1983; Paige \& Hillel, 1993), havendo necessidade de se realizar um experimento que permita a evaporação de água do perfil para obter a condutividade hidráulica para valores mais baixos de umidade. Como no experimento com evaporação a área não é coberta, o monitoramento das propriedades hidráulicas pode ser feito atéque haja a ocorrência de uma chuva, o que pode limitar o período de col eta dos dados.

Além das vantagens inerentes ao $\mathrm{MPI}$, o procedimento com evaporação permite ampliar a faixa de val ores de umidade em camadas próximas à superfície, ou seja, desde condições mais úmidas até condições mais secas, em um período de tempo bem menor que o necessário, quando se depende apenas da drenagem.

Queiroz et al. (1999), num experimento com evaporação, utilizaram oMPI eobtiveram resultados deK para a profundidade de 0,25 mem uma pequena faixa de umidade $\left(0,23\right.$ a $\left.0,25 \mathrm{~m}^{3} \mathrm{~m}^{-3}\right)$. Esses autores concluíram ser necessário um tempo maior de observações, para que haja mais evaporação deágua no perfil e, conseqüentemente, obter valores de condutividade em umidades menores.

Quandose utiliza o MPI com evaporação, ocorrem dois fluxos um ascendente na parte superior do perfil, que se encontra sob a ação da evaporação, e um fluxo descendente na parte mais profunda, que compõea drenagem. A profundidadelimítrofeentre o fluxo ascendente e o descendente é denominada profundidade do plano de fluxo zero ( $\left.z_{\mathrm{PF}}\right)$, na qual não há movimento de água.

Além das dificuldades para determinar simultaneamente o perfil de potenciais e a variação temporal da armazenagem da água no solo, comuns em experimentos de perfil instantâneo e descritos em diversos trabalhos (Baker et al., 1974; Bouma, 1983; Paige \& Hillel, 1993; J ong van Lier, 2001), quando se aplica o método com evaporação acrescenta-se a necessidade da determinação de ZPFZ em função do tempo.

U ma vez que não se encontra na literatura um método para a anál ise de resultados de experimentos de perfil instantâneo com evaporação, o presente trabalho teve por finalidade propô-lo e apresentar um exemplo de sua aplicação.

\section{MÉTODOS}

\section{Fundamentos teóricos}

O MPI baseia-sena determinação da condutividade hidráulica do solo pela quantificação dos componentes da equação de Richards, em condições transientes:

$$
\frac{\partial \theta}{\partial \mathrm{t}}=\frac{\partial}{\partial \mathrm{z}}\left(\mathrm{K}\left(\theta, \psi_{\mathrm{m}}\right) \frac{\partial \psi_{\mathrm{t}}}{\partial \mathrm{z}}\right)
$$

em que $\theta\left(\mathrm{m}^{3} \mathrm{~m}^{-3}\right)$ é a umidade volumétrica, $\mathrm{t}(\mathrm{h})$ é o tempo, $K\left(\theta, \psi_{m}\right)\left(m^{-1}\right)$ éa condutividade hidráulica em função da umidade ou do potencial matricial, $\psi_{\mathrm{t}}$ $(\mathrm{m})$ é o potencial total da água e $z(\mathrm{~m})$ éa coordenada vertical de posição.

Considerando um solo no qual há uma zona de fluxo ascendente na parte superior do perfil e outra de fluxo descendente na parte inferior, separadas por um plano de fluxo zero (PFZ), e integrando-se a equação de Richards (Equação 1) com relação à profundidade, entre a profundidade do plano de fluxo zero $\left(z_{\mathrm{PFZ}}, \mathrm{m}\right)$ e outra profundidade $\mathrm{z}(\mathrm{m})$, com as condições de contorno:

$$
\begin{gathered}
z=z_{P F Z} ; t>0 ; q=0 \\
z=z ; t>0 ; q=q_{z}
\end{gathered}
$$

obtém-se:

$$
\int_{\mathrm{z}_{\mathrm{PFZ}}}^{\mathrm{z}} \frac{\partial \theta}{\partial \mathrm{t}} \mathrm{dz}=\left.\mathrm{K}\left(\theta, \psi_{\mathrm{m}}\right) \frac{\partial \psi_{\mathrm{t}}}{\partial \mathrm{z}}\right|_{\mathrm{z}}=\mathrm{q}_{\mathrm{z}}
$$

ou, rearranjando

$$
\mathrm{K}\left(\theta, \psi_{\mathrm{m}}\right)_{\mathrm{Z}}=\frac{\mathrm{q}_{\mathrm{Z}}}{\left.\frac{\partial \psi_{\mathrm{t}}}{\partial \mathrm{z}}\right|_{\mathrm{Z}}}=\frac{\int_{\mathrm{Z}_{\mathrm{PFZ}}}^{\mathrm{z}} \frac{\partial \theta}{\partial \mathrm{t}} \mathrm{dz}}{\left.\frac{\partial \psi_{\mathrm{t}}}{\partial \mathrm{z}}\right|_{\mathrm{Z}}}=\frac{\frac{\partial \mathrm{h}_{\mathrm{Z}_{\mathrm{PFZ}}-\mathrm{z}}}{\partial \mathrm{t}}}{\left.\frac{\partial \psi_{\mathrm{t}}}{\partial \mathrm{z}}\right|_{\mathrm{Z}}}
$$

em que $h_{z^{P F z}}-z\left(m^{3} m^{-2}\right)$ é a armazenagem de água no sol o entre a profundidade do plano de fluxo zero $\left(z=z_{\mathrm{PFZ}}\right)$ ea profundidade $z$.

\section{Experimento de campo}

O experimento de campo consiste normalmente no monitoramento de instrumentos de medição do potencial matricial da água no solo (tensiômetros, tensímetros) e, ou, da umidade do solo (TDR, sonda de nêutrons) em algumas profundidades ao longo do tempo, partindo de uma condiçãoúmida imposta por uma chuva ou irrigação. No caso de haver apenas a medição de $\psi_{\mathrm{m}}$ ou $\theta$, e não ambas, a curva de retenção deve ser determinada para permitir o processamento dos dados.

\section{Estimativa da posição do plano de fluxo zero}

No PFZ, por definição, o fluxo é zero e, pela Lei de Darcy, $\partial \psi_{t} / \partial z=0$. Diversos experimentos mostraram que uma equação pol inomial de segundo grau com parâmetros de ajuste a $(\mathrm{m}), \mathrm{b}$ e c $\left(\mathrm{m}^{-1}\right)$ se ajusta geralmente bem aos dados experimentais de $\psi_{\mathrm{t}}$ versus $\mathrm{z}$, de forma que:

$$
\psi_{\mathrm{t}}=\mathrm{a}+\mathrm{bz}+\mathrm{cz} \mathrm{z}^{2}
$$


e, sendo assim:

$$
\nabla \psi_{\mathrm{t}}=\frac{\mathrm{d} \psi_{\mathrm{t}}}{\mathrm{dz}}=\mathrm{b}+2 \mathrm{cz}
$$

I gualandoa Equação (5) a zero, obtém-se quezpFz é dado por:

$$
\mathrm{z}_{\mathrm{PFZ}}=-\frac{\mathrm{b}}{2 \mathrm{c}}
$$

Como o PFZ pode mudar de posição ao longo do tempo - a tendência comum é a de se aprofundar com o tempo - um valor para Z ZFz será obtido pela Equação (6) para cada tempo de observação experimental.

Para permitir interpolações e, ou, para suavizar o conjunto de dados obtidos, os valores dos dados estimados de $Z_{\text {pFz, }}$ para cada tempo (t) de observação, podem ser ajustados a uma equação de ajuste.

\section{Cálculo da condutividade hidráulica}

A densidade de fluxo de água $\left(q_{z}\right.$ da E quação 3$)$ é calculada para cada profundidade e tempo de observação.

Considera-se que o instrumento de medição de umidade instalado na profundidade $z_{i}$ representa uma faixa de profundi dades entreum limite superior $\left(z_{a}\right)$ e um limite inferior $\left(z_{b}\right)$, de forma que $z_{a}=\frac{z_{i-1}+z_{i}}{2}$ e $z_{b}=\frac{z_{i}+z_{i+1}}{2}$, em que $z_{i-1}$ e $z_{i+1}$ são as profundidades de instalação imediatamente acima e abaixo de $z_{\mathrm{i}}$. Nessa faixa, para fins de cál culo da variação de armazenagem ( $\partial$ h da Equação 3), considera-se que a umidade seja igual à umidade observada em $z_{i}$. A densidade de fluxo, o gradiente do potencial total e a condutividade hidráulica são estimados para as mesmas profundidades onde se encontram instalados os instrumentos de medição.

Assim, a densidade de fluxo de água na profundidade de instalação dos instrumentos (profundidade média de cada camada) pode ser calculada como expressado na Equação (2), utilizando-se a taxa de variação da umidade pelo tempo, entre a profundidade média da camada e o ZPFZ.

A condutividade hidráulica pode ser obtida por:

$$
\mathrm{K}=\mathrm{q}_{\mathrm{Z}-\mathrm{Z}_{\mathrm{PFZ}}} / \nabla \psi_{\mathrm{t}}
$$

em que K é a condutividade hidráulica $\left(\mathrm{m} \mathrm{h}^{-1}\right)$ e $\nabla \psi$ é o gradiente de potencial total na camada $\left(\mathrm{m} \mathrm{m}^{-1}\right)$, calculado pela Equação 5. Considerou-se essa condutividade hidráulica representativa para oteor de água e o potencial matricial no tempo médio ena profundidade de observação.

\section{EXEMPLO DE APLICAÇÃO E DISCUSSÃO}

Um experimento de evaporação foi realizado entre os dias 9 e 26 de abril de 2002 (401 h), em uma área situada no campus da Escola Superior de Agricultura "Luiz de Queiroz" - ESALQ/USP em Piracicaba (SP) a $22^{\circ} 42^{\prime} 30$ " de latitude sul e $47^{\circ} 38^{\prime} 00$ " de longitude oeste, em um Latossolo Vermel ho-Amarelo (Typic Hapludox). O resumo de alguns dados meteorológicos do período do ensaio, observados no Posto Meteorológico do Departamento de Ciências Exatas da ESALQ/USP, localizado a $1 \mathrm{~km}$ da área experimental, encontra-se na figura 1. As medidas foram iniciadas após uma chuva que deixou o solo em condição muito úmida, não se observando, durante o período de monitoramento, precipitação. Nolocal de observação, encontravamseinstalados cinco tensi ômetros com manômetro de mercúrio em configuração circular, nas profundidades médias de 0,075, 0,225, 0,375, 0,525 e $0,675 \mathrm{~m}$, com a finalidade de medir o potencial matricial. A umidade foi estimada por meio da equação de van Genuchten, com os parâmetros das curvas de retenção obtidos por meio de amostras retiradas a menos de $1 \mathrm{~m}$ do local e em cada profundidade $\left(\theta_{\mathrm{s}}=0,446 \mathrm{~m}^{3} \mathrm{~m}^{-3}, \theta_{\mathrm{r}}=0,209 \mathrm{~m}^{3} \mathrm{~m}^{-3}, \alpha\right.$ $=0,26 \mathrm{~m}^{-1}, \mathrm{n}=1,496 \mathrm{em}=13,545$ ) (dados publicados por Cichota, 2003).

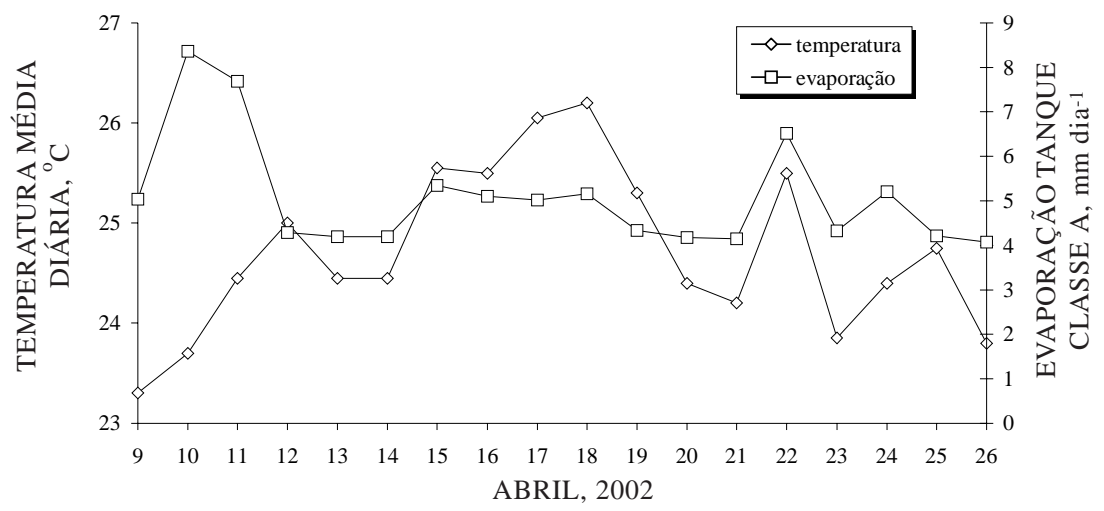

Figura 1. Temperatura média diária $\left({ }^{\circ} \mathrm{C}\right)$ e evaporação do tanque Classe $A(\mathrm{~mm} / \mathrm{dia})$ para o período de monitoramento de 9 a 26 de abril de 2002, obtidos no Posto Meteorológico do Departamento de Ciências Exatas da ESALQ/USP, Piracicaba (SP). 


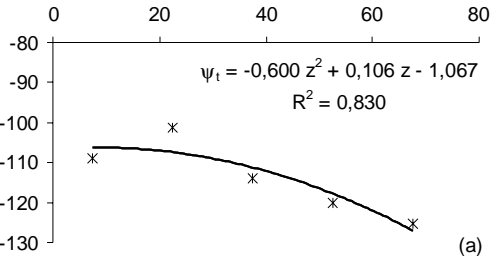

(a)

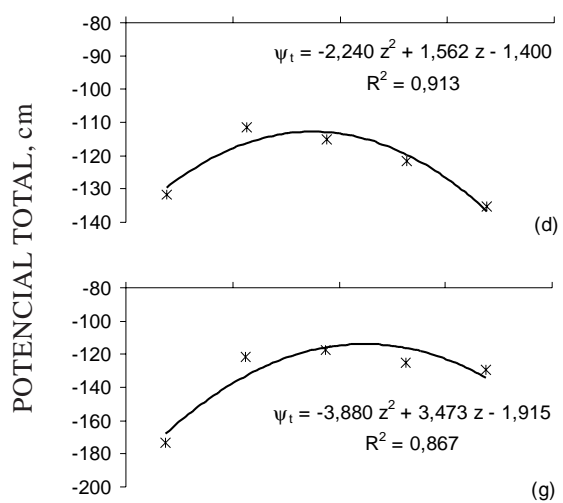

(g)

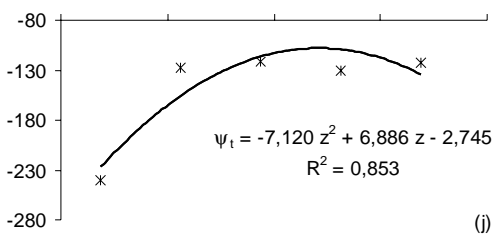

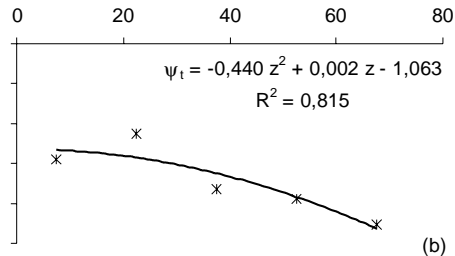
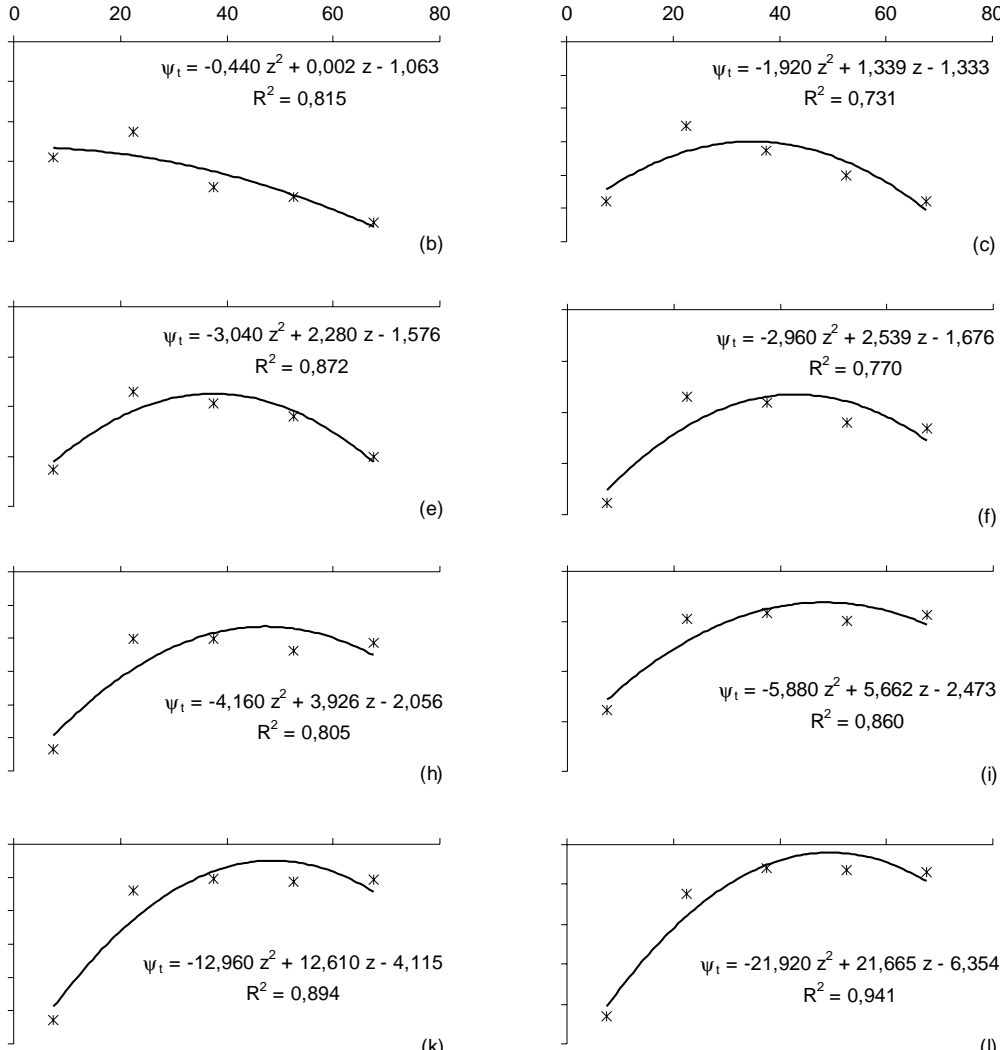

PROFUNDIDADE MONITORADA, $\mathrm{cm}$

Figura 2. Potencial total $\left(\psi_{\mathrm{t}}\right)$ em função da profundidade (z) após $0 \mathrm{~h}(\mathrm{a}), 17,9 \mathrm{~h}(\mathrm{~b}), 47,7 \mathrm{~h}(\mathrm{c}), 65,1 \mathrm{~h}$ (d), $88,5 \mathrm{~h}(\mathrm{e}), 114,4 \mathrm{~h}(\mathrm{f}), 137,8 \mathrm{~h}(\mathrm{~g}), 163,8 \mathrm{~h}(\mathrm{~h}), 186,8 \mathrm{~h}(\mathrm{i}), 211,5 \mathrm{~h}(\mathrm{j}), 282,6 \mathrm{~h}$ (k), 401,4 h (I), junto com a curva polinomial de $2^{\circ}$. grau (E quação 4 ) ajustada.

F oram feitas 12 observações notempo, cada uma resultando em um conjunto de dados $(\psi t, z)$, aos quais foi ajustada a E quação 4. Na figura 2, sãoapresentados esses conjuntos ea curva ajustada, utilizada com a finalidade de determinar não só a profundidade do plano de fluxo zero para cada tempo, mas também o gradiente de potencial total (Equação 5).

A equação polinomial utilizada apresentou um bom $R^{2}$ (mai or que 0,8 em 10 dos 12 casos) para todos os tempos monitorados, mostrando que a equação representa bem o fenômeno estudado.

Observou-se que um PFZ determinado pelo máximo das curvas ajustadas apareceu a partir de aproximadamente dois dias (Figura 2c) do início do monitoramento. Pode-se notar, nos dois primeiros tempos, um valor máximo em 0,225 m, no entanto, esse máximo não foi evidenciado pelas curvas ajustadas. Optou-se pela estimativa do PFZ pelas curvas descritas pela E quação (4), em vez de utilizar os val ores individuais, pois as curvas baseiam-senos cinco pontos observados. Além disso, a discrepância diminuiu com a profundidade de observação.

Nestetrabal ho, a equação pol inomial de segundo grau (E quação 8) com parâmetros de ajustep (m), q $\left(m h^{-1}\right)$ er $\left(m h^{-2}\right)$ foi utilizada para ajustar os dados de $z_{\mathrm{PFZ}}$, para cada tempo (t) de observação:

$$
\mathrm{z}_{\mathrm{PFZ}}=\mathrm{p}+\mathrm{qt}+\mathrm{rt}^{2}
$$

F oram estimados val ores para $Z_{\mathrm{PF} Z}$ pela E quação (6) e estes, em função do tempo, foram ajustados à Equação (8), como mostra a figura 3 para o presente exemplo, evidenciando que oPFZ se apr ofunda com o tempo e não ultrapassa a profundidade de aproximadamente $0,50 \mathrm{~m}$, semel hantemente ao encontrado por Queiroz et al. (1999).

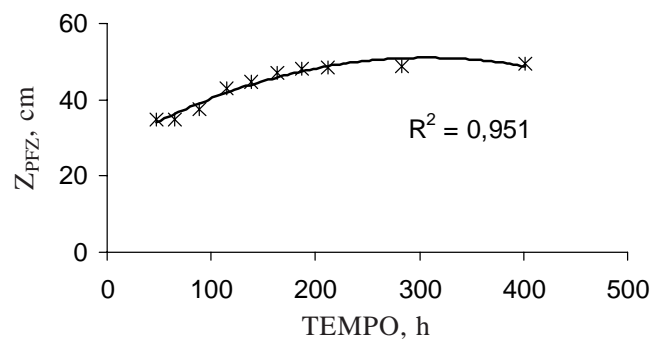

Figura 3. Profundidade do plano de fluxo zero ( $z_{\mathrm{PFZ}}$ $\mathrm{cm})$ em função do tempo $(\mathrm{h})$ e curva polinomial de segundo grau (E quação 8) ajustada. 
A partir do conhecimento do $\mathrm{Z}_{\mathrm{PF} Z}$ para qualquer tempo, o procedimento de cálculo para a obtenção da densidade de fluxo médio entre dois tempos de observação é ilustrado no esquema da figura 4. Tomando como exemplo a primeira camada do perfil de solo monitorado, ofluxo na profundidade de 0,075 $m\left(q_{z=0,075}\right)$ é cal culado por "uma das equações dessa figura".

Desse modo, a condutividade hidráulica do solo foi obtida por meio da Equação (7) para os tempos médios aos tempos de observações, encontrando-se resumi dos os val ores determi nados para a primeira camada $(0,075 \mathrm{~m})$ do experimento com evaporação (Quadro 1).

O gradientedepotencial total variou desdeval ores em torno de 0, no início do experimento, a valores superiores a 10, no final, condizente ao esperado em condições de evaporação em equilíbrio dinâmico, onde a diminuição da umidade e da condutividade hidráulica é compensada pelo aumento de $\nabla \psi_{\mathrm{t}}$. Observou-sequeapenas no primeirotempo $(\mathrm{t}=8,9 \mathrm{~h})$ a drenagem predominou sobre a evaporação, fato indicado pelo gradiente de potencial total negativo.

Observando a figura 5 , verifica-se que a faixa de umidade variou de $0,251 \mathrm{~m}^{3} \mathrm{~m}^{-3}$, quando o solo encontrava-se úmido, até $0,209 \mathrm{~m}^{3} \mathrm{~m}^{-3}$, no final do experimento. Acredita-se ser essa uma faixa considerável, uma vez que, em experimentos no mesmo local sem evaporação de água no perfil, a faixa observada para a mesma profundidade foi de 0,396, no início da drenagem, até $0,261 \mathrm{~m}^{3} \mathrm{~m}^{-3}$, no final (Berretta-Hurtado, 2004). Dessa forma, a complementação dos dados com a faixa de umi dade observada sob evaporação contribui para um aumento significativo da faixa de umidade avaliada.

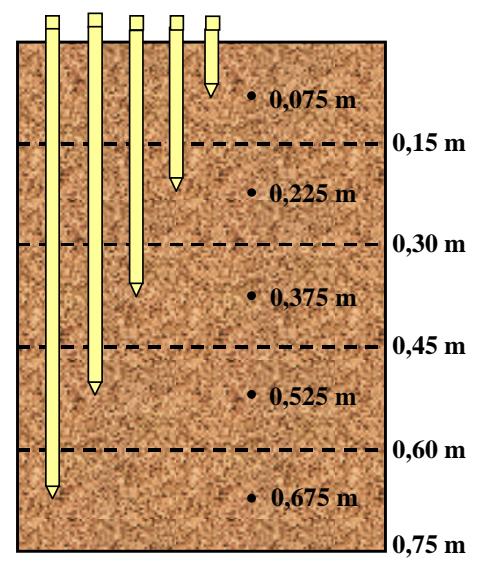

$$
\begin{aligned}
& \text { Se } 0,075<z_{P F Z}<0,15: \\
& q_{1}=\frac{d \theta_{1}}{d t}\left(z_{P F Z}-0,075\right) \\
& \text { Se } 0,15<z_{P F Z}<0,30: \\
& q_{1}=\frac{d \theta_{1}}{d t}(0,075)+\frac{d \theta_{2}}{d t}\left(z_{P F Z}-0,15\right) \\
& \text { Para } 0,30<z_{P F Z}<0,45: \\
& q_{1}=\frac{d \theta_{1}}{d t}(0,075)+\frac{d \theta_{2}}{d t}(0,15)+\frac{d \theta_{3}}{d t}\left(z_{P F Z}-0,30\right) \\
& \text { Para } 0,45<z_{P F Z}<0,60: \\
& q_{1}=\frac{d \theta_{1}}{d t}(0,075)+\frac{d \theta_{2}}{d t}(0,15)+\frac{d \theta_{3}}{d t}(0,15)+ \\
& +\frac{d \theta_{4}}{d t}\left(z_{P F Z}-0,45\right)
\end{aligned}
$$

Figura 4. Desenho esquemático do perfil de solo, com as cinco profundidades monitoradas.

Quadro 1. Valores estimados da profundidade do plano de fluxo zero, densidade de fluxo deágua e gradiente de potencial total para os tempos médios aos tempos de monitoramento, na camada de 0,075 m.

\begin{tabular}{cccr}
\hline $\mathbf{t}$ & $\mathbf{Z P F Z}$ & $\mathbf{q}_{\mathbf{0 , 0 7 5}}-\mathbf{Z}_{\mathbf{P F Z}}$ & $\nabla \mathbf{} \mathbf{t}$ \\
\hline $\mathrm{h}$ & $\mathrm{m}$ & $\mathrm{m} \mathrm{h}-1$ & $\mathrm{~m} \mathrm{~m}^{-1}$ \\
8,9 & 0,287 & $-1,01 \times 10^{-3}$ & $-0,024$ \\
32,8 & 0,321 & $-2,63 \times 10^{-4}$ & 0,494 \\
56,4 & 0,352 & $-1,50 \times 10^{-4}$ & 1,139 \\
76,8 & 0,377 & $-1,09 \times 10^{-4}$ & 1,525 \\
101,4 & 0,404 & $-8,16 \times 10^{-5}$ & 1,960 \\
126,1 & 0,428 & $-6,51 \times 10^{-5}$ & 2,493 \\
150,8 & 0,449 & $-5,41 \times 10^{-5}$ & 3,097 \\
175,3 & 0,467 & $-4,62 \times 10^{-5}$ & 4,041 \\
199,1 & 0,481 & $-4,05 \times 10^{-5}$ & 5,299 \\
247,0 & 0,501 & $-3,24 \times 10^{-5}$ & 8,242 \\
342,0 & 0,507 & $-2,31 \times 10^{-5}$ & 14,521 \\
\hline
\end{tabular}

Os valores de $\mathrm{K}$ versus $\theta$ determinados para os mesmos tempos são representados na figura 5 , conjuntamente com uma equação exponencial ajustada.

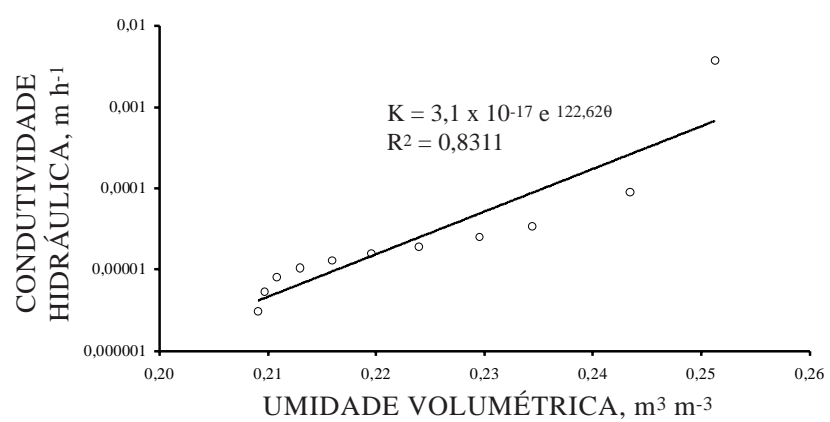

Figura 5. Condutividade hidráulica em função da umidade volumétrica (pontos), estimada com base num experimento com evaporação, para a profundidade de $0,075 \mathrm{~m}$, conjuntamente com equação exponencial ajustada. 


\section{CONCLUSÕES}

1. Equações polinomiais de segundo grau ajustaram-se bem aos conjuntos de dados de potencial total versus profundidadee da profundidade do plano de fluxo zero e o tempo.

2. Os resultados experimentais apresentados, analisados da forma proposta, indicaram a viabilidade do método, útil especialmente para se estender a faixa de umidade obtida em tais experimentos na camada superficial.

\section{LITE RATURA CITADA}

BAKER, F.G.; VENEMAN, P.L.M. \& BOUMA, J. Limitations of the instantaneous profile method for field measurement of unsaturated hydraulic conductivity. Soil Sci. Soc. Am. Proc., 38:885-888, 1974.

BERRETTA-HURTADO, A.L. Variabilidade da condutividade hidráulica do sol o em val ores fixos de umidadee de potencial matricial. Piracicaba, Escola Superior de Agricultura "Luiz de Queiroz", 2004. 94p. (Tese de Doutorado)

BOUMA, J. Use of soil survey data to select measurement techniques for hydraulic conductivity. Agric. Water Maneg., 6:177-190, 1983.

CICHOTA, R. Avaliação no campo de um TDR segmentado para determinação da umidade do solo. Piracicaba, Escola Superior de Agricultura "Luiz de Queiroz", 2003. 100p. (Tese de Mestrado)

HILLEL, D.; KRENTOS, V.D. \& STYLIANOU, Y. Procedure and test of an internal drainage method for measuring soil hydraulic characteristics in situ. Soil Sci., 114:395-400, 1972.

J ONG VAN LIER, Q. Análise de sensibilidade de um al goritmo computacional para processamento de dados de experimentos de perfil instantâneo. Piracicaba, Escola Superior deAgricultura “Luiz de Queiroz", 2001. 73p. (Tese de Livre Docência)
J ONG VAN LIER, Q. \& LIBARDI, P. Variabilidade dos parâmetros da equação que relaciona a condutividade hidráulica com a umidade do solo no método do perfil instantâneo. R. Bras. Ci. Solo, 23:1005-1014, 1999.

KLUTE, A. \& DIRKSEN, C. Hydraulic conductivity and diffusivity: Laboratory methods. In: KLUTE, A., ed. Methods of soil analysis. Madison, Soil Science Society of America, 1986. p.697-734.

LIBARDI, P. Dinâmica da água no solo. 2.ed. Piracicaba, P.L. Libardi, 2000. 509p.

MARION, J .M.; OR, D.; ROLSTON, D.E.; KAVVAS, M.L. \& BIGGAR, J .W. Evaluation of methods for determining soilwater retentivity and unsaturated hydraulic conductivity. Soil Sci., 158:1-13, 1994.

OGATA, G. \& RICHARDS, L.A. Water content changes following irrigation of bare-field soil that is protected from evaporation. Soil Sci. Soc. Am. Proc., 21:355-356, 1957.

PAIGE, G.B. \& HILLEL, D. Comparison of three methods for assessing soil hydraulic properties. Soil Sci., 155:175-189, 1993.

PREVEDELLO, B.M.S.; PREVEDELLO, C.L. \& LIBARDI, P.L. Simplificação analítica do método do perfil instantâneo para obtenção da condutividade hidráulica não saturada em condições de campo. R. Bras. Ci. Solo, 5:93-97, 1981.

QUEIROZ, M.M.F.; RUSTEBERG, B.; GHEYI,H.R. \& GUERRA, H.O.C. Estudo da condutividade hidráulica de um solo aluvial em lisímetros. R. Bras. Eng. Agric. Amb., 3:161166, 1999.

SHOUSE, P.J .; SISSON, J .B.; ELLSWORTH, T.R. \& J OBES, $J$.A. Estimating in situ unsaturated hydraulic properties of vertically heterogeneous soils. Soil Sci. Soc. Am. Proc., 56:1673-1679, 1992.

WARRICK, A.W. \& NIELSEN, D.R. Spatial variability of soil physical properties in the field. In: HILLEL, D., ed. Applications of soil physics. New York, Academic Press, 1980. p.319-344.

WATSON, K.K. An instantaneous profile method for determining the hydraulic conductivity of unsaturated porous materials. Water Res. Res., 2:709-715, 1966. 
Ana Lúcia Berretta Hurtado et al.

R. Bras. Ci. Solo, 29:301-307, 2005 